\title{
Generalization of respiratory symptom triggers
}

Thomas Janssens, Farah Martens, Nathalie Storms, \& Omer Van den Bergh

Research Group on Health Psychology, KU Leuven, Leuven, Belgium

Keywords: generalization; category learning; physical symptoms; triggers; respiration

Corresponding Author:

Thomas Janssens

Research Group on Health Psychology

KU Leuven

Tiensestraat 102 - Box 3726

3000 Leuven

Belgium

$+32(0) 16373009$

Thomas.janssens@ppw.kuleuven.be 


\begin{abstract}
:
Behavioral management of asthma and other chronic conditions depends upon the accurate identification of environmental factors that trigger symptom onset. In this study, we developed a lab-based conditioning method to study category-based acquisition and generalization of respiratory symptom triggers. During trigger acquisition, unique exemplars of two different categories were shown to a sample of healthy participants $(n=48)$. $C S+$ exemplars were paired with $\mathrm{CO}_{2}$ inhalation on $50 \%$ of trials, whilst CS- exemplars were always paired with room air. Trigger categories differed in their conceptual similarity. In a generalization task, participants rated symptom expectancy for a set of triggers that included previously seen exemplars, novel exemplars, and exemplars from novel categories. Results show that participants acquired differential symptom expectancies based on category information, which generalized to novel CS+ exemplars and novel categories that shared similarity with the CS+ category. Greater similarity between CS+ and CS- categories increased differential effects for both old and novel exemplars of CS+ and CS- categories, and increased the proportion of novel CS+ exemplars that were remembered as being seen during acquisition. These findings suggest that a more narrowly defined contrast between triggers and non-triggers promotes category-based inference and could help to reduce uncertainty about potential triggers.
\end{abstract}


Asthma is a chronic respiratory disorder that is characterized by airway inflammation, intermittent airway constriction and physical symptoms such as shortness of breath and cough (Global Initiative for Asthma (GINA), 2012). Worldwide, asthma affects about 300 million individuals (GINA, 2012). In the United States, the overall prevalence of asthma is $7.8 \%$, but prevalence is markedly higher amongst members of ethnic minorities and individuals living in poverty (Moorman, Zahran, Truman, \& Molla, 2011).

As a chronic condition, asthma cannot be cured. However, the symptoms of asthma can be controlled by taking appropriate medication and by identifying and avoiding environmental exposures that may trigger asthma symptoms (GINA, 2012). Asthma guidelines state that asthma is best managed collaboratively by the patient and health care provider, and put an emphasis on training asthma self-management skills as part of this collaboration (GINA, 2012).

Asthma symptoms can occur in response to a variety of environmental triggers and the number and type of triggers may differ greatly among individuals. This makes asthma trigger identification and avoidance important aspects of asthma management (Janssens \& Ritz, 2013). However, in day-to-day care, systematic identification of asthma triggers is often lacking and trigger management options are only briefly (if at all) discussed by health care practitioners (Rank, Wollan, Li, \& Yawn, 2010). Therefore, it is not surprising that individuals with asthma indicate a lack of knowledge about their asthma triggers and associated uncertainty and anxiety (Caress, Luker, Woodcock, \& Beaver, 2002; Tumiel-Berhalter \& Zayas, 2006). Furthermore, there is an emerging literature showing that the identification of asthma triggers is prone to errors. For example, in individuals with asthma, symptom beliefs often do 
not match the results of allergy skin test (Li, Andrist, Bamlet, \& Wolter, 2000; Ritz, Steptoe, Bobb, Harris, \& Edwards, 2006).

Challenges in identifying symptom triggers are not unique to asthma, but also occur in other chronic conditions such as chronic migraine, food allergy/hypersensitivity and Multiple Chemical Sensitivity (MCS) (Devriese et al., 2004; Kelman, 2007; Pereira et al., 2005). When objective symptom markers are available (e.g. double blind food challenges in food allergy/hypersensitivity), individuals that report these conditions have shown a marked inaccuracy in identifying symptom triggers (Martin \& MacLeod, 2009; Niestijl Jansen et al., 1994). The identification of symptom triggers can be conceptualized as a contingency learning task that occurs in a motivational context (identification of danger and safety). This is highly similar to the identification of cue-outcome contingencies during fear learning. Furthermore, a lack of accurate detection of danger and safety cues has been proposed as a key feature of anxiety disorders such as panic disorder and generalized anxiety disorder (Britton, Lissek, Grillon, Norcross, \& Pine, 2011; Grupe \& Nitschke, 2013; Lissek et al., 2009), for which individuals with asthma show an increased prevalence compared to healthy controls (Katon, Richardson, Lozano, \& McCauley, 2004; Lavoie, Boudreau, Plourde, Campbell, \& Bacon, 2011). Due to this high degree of similarity between fear learning and trigger identification, adapting constructs and procedures from fear learning research may be helpful in increasing our understanding of why inaccuracies in the perception of trigger-outcome contingencies occur.

A process that may help to understand inaccuracies in the identification of trigger-symptom contingencies is generalization of learned cue-outcome contingencies to similar cues. Generalization is the tendency to exhibit a conditioned 
response to a stimulus that resembles a conditioned stimulus $(\mathrm{CS}+)$, in this case a symptom trigger along a perceptual dimension such as size, shape or color (Lissek et al., 2008; Riccio, Ackil, \& Burch-Vernon, 1992). The strength of the response tends to decline as the resemblance between the stimulus and the CS+ decreases (Riccio et al., 1992). Furthermore, generalization also occurs along more abstract dimensions, such as emotional valence (Devriese et al., 2000), and recent research has highlighted the role of higher order cognitions such as conceptual knowledge or category membership as a basis for fear generalization (Dunsmoor \& Murphy, 2015). In order to explore the role of category information in fear learning, Dunsmoor and colleagues have developed a conditioning procedure that uses unique category exemplars during acquisition (Dunsmoor, Martin, \& LaBar, 2012). In this procedure, exemplars from one category (e.g. animals) are paired with unpleasant electrical stimulation for half of the exemplar presentations, whereas exemplars from another category (e.g. tools) are never paired with the electrical stimulation. Crucially, during the experiment, exemplars are never repeated, so in order to learn about the predictive value of $\mathrm{CS}+$ exemplars, participants have to rely on the shared category relationship of CS+ exemplars. Using this procedure, Dunsmoor and colleagues have shown that category information is used to generate shock expectancy and conditioned fear responses for novel category exemplars and that recognition rates improved for CS+ exemplars compared to CS- exemplars (Dunsmoor et al., 2012).

In asthma trigger identification, generalization effects could explain why patients that have experienced respiratory symptoms in response to a specific asthma trigger may expect the same symptoms to occur when confronted with asthma triggers that resemble the original trigger. For example, an allergic response to the neighbor's cat might lead an asthma patient to expect symptoms when confronted with other pets 
or animals. The ability to learn which stimulus forms a threat and to generalize this information to stimuli that are very much alike is a useful survival mechanism. However, widespread generalization may cause patients to unnecessarily avoid a wide variety of triggers.

In anxiety disorders, category-based generalization has been described as a characteristic of phobias. Individuals with arachnophobia, for example, not only fear spiders but also different situations (seeing a web, going into a basement) that have the same consequences (presence of a spider) and thus belong to the same category (Shepard, 1987). Indeed, recent research has shown that category membership promotes generalization of threat avoidance in phobic individuals (Dymond, Schlund, Roche, \& Whelan, 2013). Similarly, individuals with obsessive-compulsive disorder tend to avoid a wide range of cues that share a common association with danger or harmfulness (Hermans, Baeyens, \& Vervliet, 2013).

In this study, we explored the role of generalization of asthma triggers as a mechanism for the development of inaccurate asthma trigger beliefs. Using a laboratory-analog design, we explored the acquisition of trigger beliefs for different categories of asthma triggers and the generalization of asthma trigger beliefs to novel categories of varying similarity. Furthermore, we investigated category similarity and timing of the generalization test as potential moderators of the generalization of acquired trigger beliefs to novel symptom triggers, and memory for trigger exemplars. 


\section{Methods}

\section{Participants}

Participants were 50 healthy individuals ( 9 men and 41 women, age $=18-28$ years). Eighteen of them were undergraduate students who received course credit, the other volunteers received $€ 15$ as compensation. Exclusion criteria were poor lung function (forced expiratory volume in 1 second (FEV1) that was less than $80 \%$ of the predicted value), a self-reported diagnosis of asthma, allergies, cardiac and pulmonary diseases, epilepsy, clinical depression, anxiety disorders or other serious medical conditions. Two female participants were excluded on the basis of these criteria, resulting in a final sample of forty-eight participants. The study was approved by the Multidisciplinary Ethical Committee of the Faculty of Psychology and Educational Sciences of the University of Leuven.

\section{Materials and apparatus}

Self-report measures. Participants rated their symptom expectancy on a horizontal visual analog scale (VAS). The scale ranged from 0 (definitely do not expect) to 100 (definitely expect). Symptom intensity and symptom unpleasantness was measured using 2 horizontal VAS scales with labels "not at all intense" and "maximal imaginable intensity" cq. "not at all unpleasant" and "maximal imaginable unpleasantness". These VAS scales were presented on a computer screen. Valence and arousal of the trials were measured using a paper-based version of the selfassessment MANIKIN scales (Bradley \& Lang, 1994). Both scales are 9-point pictorial scales. Valence anchors are Pleasant (scored as 1) and Unpleasant (scored as 9). Arousal anchors are Exited (scored as 1) and Calm (scored as 9). A posteriori trigger expectancies were measured in an online survey in which participants 
indicated for every asthma trigger (a) whether they had seen it during the experiment (yes/no) and (b) whether they expected to experience respiratory symptoms when confronted with this trigger. Expectancy was measured on a scale from 0 (do not expect respiratory symptoms at all) over 5 (don’t know) to 10 (certainly expect respiratory symptoms).

Apparatus. Participants either breathed regular room air or an air mixture containing $7.5 \% \mathrm{CO}_{2}, 21 \% \mathrm{O} 2$ and $71.5 \% \mathrm{~N}_{2}$. The $\mathrm{CO}_{2}$-enriched air was fed into a meteorological balloon. The balloon was connected to a three-way valve, which was used to switch between the $\mathrm{CO}_{2}$-enriched air from the balloon and regular room air. Participants breathed into a mask that was mounted onto the three-way valve through a bacterial filter. An infrared $\mathrm{CO}_{2}$ monitor (POET II, Criticare, Waukesha, WI) was connected to the mask via a vinyl tube to continuously sample air and monitor fractional end-tidal $\mathrm{CO}_{2}$ concentration $\left(\mathrm{Fet} \mathrm{CO}_{2}\right)$. This capnograph was calibrated daily using a gas containing $7.5 \% \mathrm{CO}_{2}$. The mask was also connected to a heated pneumotachograph (Fleisch No. 2, fg-deutschland, Hechingen, Germany) with carrier-demodulator (CD15, Validyne, Northridge, CA), which was calibrated daily using a 11 syringe. Aerosols were created using an isotonic saline solution that was vaporized at a constant airflow of 1 1/min using a nebulizer (646, DeVilbiss, Somerset, PA). The aerosol was led to the mask by a small vinyl tube. Signals from the pneumotachograph and capnograph were sampled through a NI PCI-6221 16-Bit data acquisition card (National Instruments, Austin, TX). Data were stored with Affect 4.0 software (Spruyt, Clarysse, Vansteenwegen, Baeyens, \& Hermans, 2010), which was also used for stimulus presentation. 
Stimuli and variables. Stimuli consisted of four categories of potential asthma triggers: mammals, birds, flowers and molds. For each trigger category 20 exemplar pictures were selected that are uncommon in Western Europe. These exotic exemplars were used in order to prevent participants from having prior knowledge concerning their potential sensitivity to these specific triggers. Categories were selected so that they could be organized based on conceptual similarity: mammals being more similar to birds compared than to flowers or molds, and flowers being more similar to molds than to birds or mammals ${ }^{1}$.

Each participant was presented with a random sample of 20 pictures that were exemplars of 2 categories: $10 \mathrm{CS}+$ pictures and $10 \mathrm{CS}$ - pictures. Participants were counterbalanced so that half of the participants received exemplars from categories that were conceptually more similar (e.g. mammals (CS+) and birds (CS-)) and the other half received exemplars from categories that were conceptually less similar (e.g. birds (CS+) and molds (CS-)). Depending on the categories that were presented during acquisition, generalization categories consisted either of categories that were unrelated to the $\mathrm{CS}$ categories $(\mathrm{Gu})$, or of categories that were conceptually more similar to the CS+ category $\left(\mathrm{G}^{+}\right)$or the CS- category (G-), cf. Table 1 for an overview. During the generalization phase, CS+ and CS- triggers were further subdivided into exemplars that had been presented during the acquisition trial and novel CS+/CS- exemplars.

\footnotetext{
${ }^{1}$ Category similarities were verified in a separate sample $(n=35)$. Participants were presented with all pairwise combinations of category labels and rated similarities of categories. A planned comparison (Mammals:Birds and Flowers:Molds) vs. (Mammals:Molds, Mammals:Flowers, Birds:Molds, and Birds:Flowers) showed that similarities were greater for the "similar" categories compared to the "different" categories $(\mathrm{F}(1,34)=99.7, \mathrm{p}<.0001)$
} 


\section{Procedure}

Lab session. Participants received the information that they were to inhale a series of 20 aerosols, each containing a mixture of air and an artificially produced allergen of a specific animal or plant. During each breathing trial, they would be able to see a picture of this specific animal or plant on a computer screen. Participants were warned that they might experience some respiratory complaints during the breathing trials, like shortness of breath, chest tightness, irritation of the throat, lightheadedness, faster heartbeat, tendency to breathe faster or other symptoms. Participants' sensitivity to the different allergens would be monitored. They were allowed to stop the experiment at any time.

Following completion of the informed consent, lung function was measured with the use of a spirometer (Jaeger Masterscope: Hoechberg, Germany). Subsequently, the experimenter left the room and breathing trials started. Each of the 20 breathing trials had the same structure. First the picture of a trigger was shown. The CS+ and CS- pictures were presented in randomized order. Half of the CS+ images were followed by inhalation of $\mathrm{CO}_{2}$-enriched air (Uncondtioned Stimulus, UCS); the remaining images were all followed by inhalation of regular room air through the mask. For 24 participants, the CS+ and CS- trigger categories were more similar, whilst for the other half of the participants the CS+ and CS- triggers were less conceptually similar. After $5 \mathrm{~s}$ of trigger presentation, participants rated their expectancy to experience symptoms when breathing an aerosol containing the allergen of this trigger. The trigger picture remained visible until participants had completed the expectancy rating. Next, participants were prompted to breathe through 
the mask. Throughout the trial, the picture of the trigger remained visible. After 60 seconds participants were allowed to take off the mask, and a recovery phase of 2 minutes began. At the start of the recovery phases, participants rated the intensity and the unpleasantness of symptoms they might have felt during the breathing trial. When the two minutes had passed, participants were able to start a new breathing trial by pressing the space bar. After completion of all 20 breathing trials, participants received a flyer with the link to the online survey and the date on which they had to fill out the survey. The total duration of the lab session was approximately 90 minutes.

Online survey. Participants were asked to complete the online survey either one day or one week after the lab session. In this survey, participants indicated for the full set of 80 triggers (20 triggers exemplars per category) whether they had seen the trigger during the lab session, and rated symptom expectancy for each of the triggers. Triggers were presented in randomized order and participants completed ratings at their own pace. During this phase, participants did not breathe through a mouthpiece and there were no UCS presentations. Participants were fully debriefed after completion of the survey.

\section{Data reduction and analysis}

Data from the pneumotachograph and capnograph were processed using PSPHA (De Clerck, Verschuere, Crombez, \& De Vlieger, 2006), resulting in calculations of time and volume parameters and the fractional concentration of endtidal $\mathrm{CO}_{2}\left(\mathrm{FetCO}_{2}\right)$ on a breath by breath basis. Based on these data, a single average of minute ventilation and $\mathrm{FetCO}_{2}$ was calculated for each acquisition trial. 
Breathing data, symptom ratings and expectancy ratings were analyzed using mixed model (hierarchical linear model) analysis. Mixed model analysis takes into account within-participant variability and allows for flexibility with regard to unbalanced designs (unequal number of observations per variable) and missing data. Continuous variables were mean centered prior to inclusion in the analyses. Data of the acquisition task were analyzed by fitting a mixed model that included fixed and random effects of CS type and trial, in order to model individual differences in learning trajectories.

A similar approach was taken for symptom ratings during the acquisition task and for the expectancy ratings during the online generalization task. These models included a fixed effect of trial type and a random intercept to allow for individual variability. Within these models we investigated generalization and safety learning using a priori contrasts, and Bonferroni-corrected post-hoc comparisons.

Recognition data for each participant were coded as hits, misses, correct rejections and false alarms for the $\mathrm{CS}+$ and CS- trigger category. Hits were defined as asthma triggers that were correctly identified as being displayed during the lab session, whereas asthma triggers that were reported as "seen" although they were not presented, were labeled false alarms. These data were used in a logistical mixed model to evaluate individual differences in stimulus recognition. All analyses were conducted in SPSS 22, using Satterthwaite approximation for degrees of freedom (IBM Corp., Armonk, NY). 


\section{Results}

\section{Trigger acquisition}

Over the course of the acquisition trials, participants showed a clear learning effect. Although symptom expectancies increased both for CS + and CS- exemplars (Trial $\mathrm{F}(1,48)=5.58, \mathrm{p}=.022)$, this increase was greater for CS+ exemplars compared to CS- symptom expectancies (CS Type $\mathrm{x}$ Trial $\mathrm{F}(1,816)=7.81, \mathrm{p}=.005)$, cf. Figure 1 . Planned comparison of CS + and CS- exemplars during the final acquisition trial showed a difference in symptom expectancy of 11 points (0-100 scale, $95 \%$ CI 5.416.7). A model that included similarity between CS+ and CS- triggers (sharing vs. not sharing a close conceptual relationship) did not show significant effects of conceptual similarity on the acquisition of symptom expectancies (CS Relationship x CS Type x Trial $F(1,816)=0.204, p=.652)$.

\section{Symptom perception and breathing behavior during acquisition}

An overview of differences in symptom perception and breathing behavior is listed in Table 2. Symptoms during $\mathrm{CO}_{2}$ trials were rated as more intense and more aversive compared to symptoms during room air trials. Breathing behavior followed a similar pattern: increased minute ventilation and $\mathrm{FetCO}_{2}$ during $\mathrm{CO}_{2}$ trials compared to room air trials. On the MANIKIN scales participants reported feeling more unpleasant and less calm during $\mathrm{CO}_{2}$ trials compared to room air trials. Furthermore, participants felt less calm (more aroused) when room air trials were preceded by a $\mathrm{CS}+$ exemplar instead of a CS- exemplar $(\mathrm{t}(854)=2.10, \mathrm{p}=.036)$. We found no effects of CS similarity on any of these variables. 


\section{Generalization of symptom expectancies to novel CS+ and CS- exemplars}

To test generalization effects, we fitted a 2 (CS type (CS+ vs. CS-)) x 2 (Novelty (old vs. new)) x 2 (CS relationship (similar vs. dissimilar)) x 2 (Timing (1 day vs. 1 week)) linear mixed model, which included a random intercept to account for within-person variability. A visual presentation of results is shown in Figure 2. The model resulted in a significant main effect of CS type $(F(1,1872)=241.66$, $\mathrm{p}<.001$ ), showing greater symptom expectancies for CS+ vs. CS- exemplars. Furthermore, there was a main effect of Novelty $(\mathrm{F}(1,1872)=9.49, \mathrm{p}=.002)$, which showed that the presentation of CS exemplars that had been seen during acquisition generated higher symptom expectancy compared to novel CS exemplars. The main effect of Timing bordered on significance $(F(1,48)=3.31, p=.075)$, with symptom expectancies being greater during generalization tests occurring 1 day after acquisition compared to one week after acquisition. These effects were further qualified by significant 2-way interactions. Further exploration of the CS type $\mathrm{x}$ Novelty $(\mathrm{F}(1,1872)=6.53, \mathrm{p}=.011)$ interaction showed that the difference between old and novel CS exemplars was only significant for CS+ exemplars $(\mathrm{p}<.001)$ and not for CS- exemplars $(p=.710)$. Exploration of the CS type x CS relationship $\mathrm{F}(1,1872)=10.42, \mathrm{p}=.001)$ showed that retention of differential acquisition effects was greater for participants that had received CS+ exemplars that were more similar $(p=.001)$. Exploration of the CS type $x$ Timing interaction $F(1,1872)=10.58, p=.001)$ showed that over time, symptom expectancy for CS+ exemplars declined $(p=.024)$, whereas there were no significant differences in symptom expectancy of CSexpectancy ratings at both time points $(\mathrm{p}=.213)$. Novelty $x$ Timing $(\mathrm{F}(1,1872)=2.96$, $\mathrm{p}=.085)$, and Novelty x CS relationship $\mathrm{F}(1,1872)=3.61, \mathrm{p}=.058)$ effects bordered on significance, and visual inspection (cf. Figure 2) suggested greater differential 
symptom expectancies after one day compared to one week, and smaller differences between old and novel CS exemplars when CSs had been more similar. There were no other significant effects (all p's >.10).

\section{Generalization to novel categories}

Generalization to novel categories was tested using a 5 (Stimulus type (CS+, G+, G-, CS-, Gu)) x 2 (Timing (1 day vs. 1 week)) linear mixed model, which included a random intercept to account for within-person variability. This resulted an a significant effect of stimulus type $(\mathrm{F}(4,3810)=120.86, \mathrm{p}<.001)$, which was further qualified by a Stimulus type $\mathrm{x}$ Timing interaction $(\mathrm{F}(4,3810)=6.28, \mathrm{p}<.001)$. Further exploration of this interaction effect showed that the generalization gradient $(\mathrm{CS}+\mathrm{G}+$ G- CS-) was steeper when generalization tests were carried out one day after trigger acquisition compared to one week after trigger acquisition $(\mathrm{t}(3792)=4.44, \mathrm{p}<.001)$, Figure 3). When tested one day after trigger acquisition, participants that had received dissimilar CS's showed a greater generalization response to generalization stimuli that shared a close conceptual relationship with the $\mathrm{CS}+$ category $(\mathrm{G}+)$, compared to generalization stimuli that shared a relationship with the CS- category (G-) $(\mathrm{t}(3792)=3.96, \mathrm{p}=0.001)$. Symptom expectancy for $\mathrm{G}+$ stimuli was greater than symptom expectancy for the same stimuli in participants for whom these stimuli were unrelated to either $\mathrm{CS}$ category $(\mathrm{Gu})(\mathrm{t}(3837)=3.30, \mathrm{p}=0.010), \mathrm{Cf}$. Figure 3 . One week after trigger acquisition, $\mathrm{CS}+$ exemplars continued to elicit symptom expectancies, but there were no longer any significant differences between G+, G-, and Gu categories (all p's >.10, cf. Figure 3).

\section{Memory for individual trigger exemplars}


Recognition of previously seen CS exemplars (hits) was not different for CS+ vs. CS-and did not differ based on CS category relationship. However, the hit rate dropped significantly $(\mathrm{p}=.002)$ from $.85[95 \%$ CI $.80-.90]$ when tested one day after acquisition to to $.71[95 \% \mathrm{CI} .62-.78]$ one week after acquisition. For false alarms, we observed a significant CS type x CS relationship interaction $(\mathrm{F}(1,953)=5.17, \mathrm{p}=.023)$ : when CS categories were more similar, false alarm rate was greater $(\mathrm{p}=.01)$ for CS+ exemplars (.18 [95\% CI .12-.26]) compared to CS- exemplars (.09 [95\% CI .06-.16]); whereas no differences in false alarm rate were observed when CS categories were more different (CS+: .11[95\% CI .06-.17]; CS-: .10[.06-.17].

For the generalization categories, overall false alarm rate was low $(0.039$, 95\%CI [0.002-0.076]), and did not differ significantly for the different types of generalization stimuli $(\mathrm{F}(2,1914)=0.41, \mathrm{p}=.663)$ or timing of the generalization tests $(\mathrm{F}(2,1914)=0.001, \mathrm{p}=.99)$. 


\section{Discussion}

In this experiment, the acquisition of differential symptom expectancies for $\mathrm{CS}+$ and CS- exemplars, as well as differential results in the recognition data and symptom expectancy ratings during the recognition task clearly indicate that participants made use of category information in order to differentiate between potential triggers of respiratory symptoms. This is in line with previous findings on the expectancy and prediction of aversive events. In fear learning, category information, derived from pre-existing conceptual relationships or the construction of novel categories, has been shown to impact generalization of fear responses (Dunsmoor et al., 2012; Dunsmoor, White, \& LaBar, 2011; Dymond et al., 2013). In the present study, we were able to show similar generalization of symptom expectancies using aversive $\mathrm{CO}_{2}$-induced respiratory symptoms as a UCS. Moreover, our experiment extends some of these previous findings by showing generalization of symptom expectancies to different categories that share a degree of similarity with the $\mathrm{CS}+$ category, and by demonstrating that generalization of symptom expectancies can be enhanced or reduced by characteristics of the learning task.

Using category exemplars that either shared or did not share a close conceptual relationship, we observed that the role of category information (e.g. exemplars from the $\mathrm{CS}+$ category are dangerous) in the generalization of symptom expectancies is dependent upon the specificity of contrasting category information. Generalization test ratings of differential expectancy between CS+ and CS- category exemplars was greater when $\mathrm{CS}+$ and CS- categories were more similar, compared to the condition where differences between CS+ and CS- categories were larger.

Previous studies have shown that perceptual, or feature-based, generalization is influenced by procedures that direct attention to relevant stimulus features, either 
by explicit instruction or by a change in the perceptual features of CS + and CS(Vervliet \& Geens, 2014; Vervliet, Kindt, Vansteenwegen, \& Hermans, 2010). These findings suggest that similar observations hold for perceptual and category-based generalization: the relevant characteristics of the $\mathrm{CS}+$ category - the characteristics that will be important for a generalized symptom expectancy to novel exemplars will depend upon the characteristics of situations in which no symptoms are experienced. The finding that participants that had received CS+/CS- exemplars that were more similar also showed an increased false alarm rate for CS+ exemplars suggests that the increased importance of category membership occurred at the expense of processing or remembering individual stimulus exemplars. The results on the recognition tasks differ from the findings of Dunsmoor et al. (2012), who did not show increased false alarm rates but observed improved hit rates for CS+ exemplars during the recognition task. However, these results are difficult to compare, as we did only find increased false alarm rates for CS+ exemplars when CS+ and CS- were related categories (a condition which was absent from Dunsmoor et al. (2012), and analysis of hit rates in Dunsmoor et al. (2012) was limited to high confidence recognition ratings.

Apart from generalization to novel CS+ exemplars, acquired category-based symptom expectancies also generalized to novel categories that were similar to the original CS categories, suggesting that previously acquired relationships among categories can be a source of generalized symptom expectancies. The observation of flattened generalization slopes with increased time since acquisition is similar to observations about generalization based on forgetting specific stimulus features (Riccio et al., 1992). However, close inspection of our results suggests that in our experiment, the flatter generalization slope is caused by a reduction of symptom 
expectancies for $\mathrm{G}+/-$ and $\mathrm{CS}-$ categories, instead of due to increased generalization, which makes this finding difficult to reconcile with a feature based approach of stimulus generalization.

These findings have important implications for the management of asthma and other chronic conditions. Based on this experiment, we belief that category based generalization of trigger beliefs has to be taken into account when informing participants about asthma triggers. We can expect that when individuals with asthma acquire beliefs about categories of triggers, these trigger beliefs can generalize to similar or related trigger categories, resulting in inaccurate identification of asthma triggers and associated unwarranted generalized trigger avoidance. Furthermore, our results suggest that one way to make trigger beliefs more specific is to provide individuals with experiences about potential triggers that are similar to the category of asthma triggers, but do not elicit symptoms. By focusing education about asthma triggers not only on the identification of potentially dangerous triggers, but also on the safety of potential triggers that are similar to the targeted asthma triggers, an intervention that is aimed at distinguishing triggers from non-triggers could contribute to a reduction of uncertainty about asthma triggers and an overall reduction in the number of triggers that participants actively have to avoid, which in turn may reduce the disease burden of asthma and other allergic conditions. Finally, actively focusing trigger education on differentiation between triggers and non-triggers may hold promises to prevent the development of trigger-related fear. Similar developments have been made in the context of internal predictors of respiratory symptoms. A CBT program with a focus on differentiation between dangerous and safe internal respiratory sensations has been successful in preventing the development of panic 
disorder in individuals with respiratory disease (Livermore, Sharpe, \& McKenzie, 2010).

A number of factors may limit our conclusions about the role of category relationship and individual risk factors in explaining differences in generalization of symptom expectancies. A first limitation is that throughout our experiment, symptom expectancy ratings were low. During acquisition, mean symptom ratings started out and remained below 50 on a $0-100$ rating scale. Several aspects of our experimental procedure could have contributed to this finding. The low number of acquisition trials $(10 \mathrm{CS}+, 10 \mathrm{CS}-)$, and limited number of $\mathrm{CO}_{2}$ trials $(50 \%$ of $\mathrm{CS}+$ trials $)$ could have played a part. However, in a similar experiment using electrocutaneous stimulation, much higher expectancy ratings were found at the end of a similar 20-trial block (Dunsmoor et al., 2012), which suggests that differences in expectancy levels between these experiments were not due to the trial structure. Instead, differences between acquisition effects may be due to differences in the type of CS's and UCS that were used. Compared to electrocutaneous stimulation, $7.5 \% \mathrm{CO}_{2}$ inhalation is characterized by sensations that are have a longer duration and a less clear onset and offset. While these characteristics add to the ecological validity of $7.5 \% \mathrm{CO}_{2}$ inhalation as a model of asthma symptoms, they may also have hindered contingency perception and longterm retention of these contingencies. Furthermore, while the UCS was standardized $\left(7.5 \% \mathrm{CO}_{2}\right)$, the perceived unpleasantness of the stimulus may have differed due to individual differences in sensitivity and breathing regulation.

The low symptom expectancy rating may also have been caused by participants' a priori trigger knowledge. Participants in our experiment did not have any allergies, which may have been associated with a strong a priori belief about a 
lack of allergic triggers. The fact that symptom expectancies at the start of the experiment were well below 50 adds to this explanation. As a priori beliefs about expectancies can play a important role in contingency perception (Alloy \& Tabachnik, 1984), it would be interesting to see if we would find similar results when selecting participants based on (an absence of) a priori trigger beliefs.

A final limitation of our study is that because only four trigger categories were used, the relationship between generalization categories and CS categories was dependent upon the relationship between CS categories, as G+ and G- categories were only available when CS+ and CS- categories did not share similarity. By including other trigger categories further research on generalization of trigger beliefs may bypass this limitation and open up opportunities to investigate the relationships between generalization to novel trigger exemplars and generalization to novel trigger categories.

In summary, we found that a conditioning procedure using unique category exemplars as CS's led participants to acquire differential symptom expectancies based on category information, and that these expectancies generalized to novel CS+ exemplars and novel categories that shared similarity with the $\mathrm{CS}+$ category. A delayed generalization test resulted in decreased retention of differential symptom expectancies and decreased generalization to novel trigger categories. Finally, a close conceptual relationship between CS+ and CS- categories increased differences between CS+ and CS- trigger expectancies effects for both old and novel category exemplars, while also increasing the false alarm rate for novel CS+ exemplars. These findings show that experiments on category-based generalization have applications in a variety of domains that are characterized by contingency learning in a motivated context. In the context of identification of asthma triggers, our findings suggest that 
focusing on the contrast between triggers and non-triggers could help to improve symptom-trigger contingency learning, and may open up opportunities to improve disease management and reduce disease burden in individuals suffering from asthma or other chronic conditions.

\section{Acknowledgements}

Dr. Janssens is a Postdoctoral Fellow of the FWO (Research foundation Flanders) Preparation of this paper was supported by the Center for Excellence on Generalization Research (GRIP*TT; KU Leuven Grant PF/10/005). 


\section{References}

Alloy, L. B., \& Tabachnik, N. (1984). Assessment of covariation by humans and animals: The joint influence of prior expectations and current situational information. Psychological Review, 91(1), 112-149. doi: 10.1037/0033295X.91.1.112

Bradley, M. M., \& Lang, P. J. (1994). Measuring emotion: the Self-Assessment Manikin and the Semantic Differential. Journal of Behavior Therapy and Experimental Psychiatry, 25(1), 49-59. doi: 10.1016/0005-7916(94)90063-9

Britton, J. C., Lissek, S., Grillon, C., Norcross, M. A., \& Pine, D. S. (2011). Development of anxiety: the role of threat appraisal and fear learning. Depression and Anxiety, 28(1), 5-17. doi: 10.1002/da.20733

Caress, A.-L., Luker, K., Woodcock, A., \& Beaver, K. (2002). An exploratory study of priority information needs in adult asthma patients. Patient Education and Counseling, 47(4), 319-327. doi: 10.1016/S0738-3991(02)00005-8

De Clerck, A., Verschuere, B., Crombez, G., \& De Vlieger, P. (2006).

Psychophysiological analysis (PSPHA): a modular script based program for analyzing psychophysiological data. Behavior Research Methods, 38(3), 504510. doi: 10.3758/BF03192805

Devriese, S., Winters, W., Diest, I., Peuter, S., Vos, G., Van de Woestijne, K., \& Van den Bergh, O. (2004). Perceived relation between odors and a negative event determines learning of symptoms in response to chemicals. International Archives of Occupational and Environmental Health, 77(3), 200-204. doi: $10.1007 / \mathrm{s} 00420-003-0488-8$

Devriese, S., Winters, W., Stegen, K., Van Diest, I., Veulemans, H., Nemery, B., . . . Van den Bergh, O. (2000). Generalization of Acquired Somatic Symptoms in 
Response to Odors: A Pavlovian Perspective on Multiple Chemical Sensitivity. Psychosomatic Medicine, 62, 751-759.

Dunsmoor, J. E., Martin, A., \& LaBar, K. S. (2012). Role of conceptual knowledge in learning and retention of conditioned fear. Biological Psychology, 89(2), 300305. doi: 10.1016/j.biopsycho.2011.11.002

Dunsmoor, J. E., \& Murphy, G. L. (2015). Categories, concepts, and conditioning: how humans generalize fear. Trends in Cognitive Sciences, 19(2), 73-77. doi: 10.1016/j.tics.2014.12.003

Dunsmoor, J. E., White, A. J., \& LaBar, K. S. (2011). Conceptual similarity promotes generalization of higher order fear learning. Learning and Memory, 18(3), 156-160. doi: 10.1101/1m.2016411

Dymond, S., Schlund, M. W., Roche, B., \& Whelan, R. (2013). The spread of fear: Symbolic generalization mediates graded threat-avoidance in specific phobia. The Quarterly Journal of Experimental Psychology, 67(2), 247-259. doi: $10.1080 / 17470218.2013 .800124$

Global Initiative for Asthma (GINA). (2012). Global Strategy for Asthma Management and Prevention. from http://www.ginasthma.org

Grupe, D. W., \& Nitschke, J. B. (2013). Uncertainty and anticipation in anxiety: an integrated neurobiological and psychological perspective. Nat Rev Neurosci, 14(7), 488-501. doi: 10.1038/nrn3524

Hermans, D., Baeyens, F., \& Vervliet, B. (2013). Generalization of Acquired Emotional Responses. In M. D. Robinson, E. R. Watkins, \& E. Harmon-Jones (Eds.), Handbook of Cognition and Emotion (pp. 117-134). New York, NY: Guilford. 
Janssens, T., \& Ritz, T. (2013). Perceived triggers of asthma: key to symptom perception and management. Clinical and Experimental Allergy, 43(9), 10001008. doi: $10.1111 /$ cea. 12138

Katon, W. J., Richardson, L., Lozano, P., \& McCauley, E. (2004). The Relationship of Asthma and Anxiety Disorders. Psychosomatic Medicine, 66(3), 349-355.

Kelman, L. (2007). The triggers or precipitants of the acute migraine attack. Cephalalgia, 27(5), 394-402. doi: 10.1111/j.1468-2982.2007.01303.x

Lavoie, K. L., Boudreau, M., Plourde, A., Campbell, T. S., \& Bacon, S. L. (2011). Association Between Generalized Anxiety Disorder and Asthma Morbidity. Psychosomatic Medicine, 73(6), 504-513. doi:

10.1097/PSY.0b013e318222e9fc

Li, J. T. C., Andrist, D., Bamlet, W. R., \& Wolter, T. D. (2000). Accuracy of patient prediction of allergy skin test results. Annals of Allergy, Asthma \& Immunology, 85(5), 382-384. doi: 10.1016/S1081-1206(10)62550-1

Lissek, S., Biggs, A. L., Rabin, S. J., Cornwell, B. R., Alvarez, R. P., Pine, D. S., \& Grillon, C. (2008). Generalization of conditioned fear-potentiated startle in humans: Experimental validation and clinical relevance. Behaviour Research and Therapy, 46(5), 678-687. doi: 10.1016/j.brat.2008.02.005

Lissek, S., Rabin, S. J., McDowell, D. J., Dvir, S., Bradford, D. E., Geraci, M., . . Grillon, C. (2009). Impaired discriminative fear-conditioning resulting from elevated fear responding to learned safety cues among individuals with panic disorder. Behaviour Research and Therapy, 47(2), 111-118. doi: 10.1016/j.brat.2008.10.017 
Livermore, N., Sharpe, L., \& McKenzie, D. (2010). Prevention of panic attacks and panic disorder in COPD. European Respiratory Journal, 35(3), 557-563. doi: $10.1183 / 09031936.00060309$

Martin, P. R., \& MacLeod, C. (2009). Behavioral management of headache triggers: Avoidance of triggers is an inadequate strategy. Clinical Psychology Review, 29(6), 483-495. doi: 10.1016/j.cpr.2009.05.002

Moorman, J. E., Zahran, H., Truman, B. I., \& Molla, M. T. (2011). Current asthma prevalence-United States, 2006-2008. MMWR. Surveillance Summaries, 60(Suppl), 84-86.

Niestijl Jansen, J. J., Kardinaal, A. F. M., Huijbers, G., Vlieg-Boerstra, B. J., Martens, B. P. M., \& Ockhuizen, T. (1994). Prevalence of food allergy and intolerance in the adult Dutch population. Journal of Allergy and Clinical Immunology, 93(2), 446-456. doi: 10.1016/0091-6749(94)90353-0

Pereira, B., Venter, C., Grundy, J., Clayton, C. B., Arshad, S. H., \& Dean, T. (2005). Prevalence of sensitization to food allergens, reported adverse reaction to foods, food avoidance, and food hypersensitivity among teenagers. Journal of Allergy and Clinical Immunology, 116(4), 884-892. doi:

10.1016/j.jaci.2005.05.047

Rank, M. A., Wollan, P., Li, J. T., \& Yawn, B. P. (2010). Trigger recognition and management in poorly controlled asthmatics. Allergy and Asthma Proceedings, 31(6), 99-105. doi: 10.2500/aap.2010.31.3405

Riccio, D. C., Ackil, J. K., \& Burch-Vernon, A. (1992). Forgetting of stimulus attributes: Methodological implications for assessing associative phenomena. Psychological Bulletin, 112(3), 433-445. doi: 10.1037/0033-2909.112.3.433 
Ritz, T., Steptoe, A., Bobb, C., Harris, A. H. S., \& Edwards, M. (2006). The Asthma Trigger Inventory: Validation of a Questionnaire for Perceived Triggers of Asthma. Psychosomatic Medicine, 68(6), 956-965. doi: 10.1097/01.psy.0000248898.59557.74

Shepard, R. (1987). Toward a universal law of generalization for psychological science. Science, 237(4820), 1317-1323. doi: 10.1126/science.3629243

Spruyt, A., Clarysse, J., Vansteenwegen, D., Baeyens, F., \& Hermans, D. (2010). Affect 4.0: A free software package for implementing psychological and psychophysiological experiments. Experimental Psychology, 57(1), 36-45. doi: $10.1027 / 1618-3169 / \mathrm{a} 000005$

Tumiel-Berhalter, L., \& Zayas, L. E. (2006). Lay experiences and concerns with asthma in an urban Hispanic community. Journal of the National Medical Association, 98(6), 875-880.

Vervliet, B., \& Geens, M. (2014). Fear generalization in humans: Impact of feature learning on conditioning and extinction. Neurobiology of Learning and Memory, 113(0), 143-148. doi: 10.1016/j.nlm.2013.10.002

Vervliet, B., Kindt, M., Vansteenwegen, D., \& Hermans, D. (2010). Fear generalization in humans: Impact of verbal instructions. Behaviour Research and Therapy, 48, 38-43. doi: 10.1016/j.brat.2009.09.005 
Table 1: Counterbalanced stimulus types

\begin{tabular}{lll}
\hline \multicolumn{3}{l}{ Close conceptual similarity between CS+ and CS- } \\
CS+ & CS- & Gu \\
Flowers & Molds & Birds, Mammals \\
Molds & Flowers & Birds, Mammals \\
Birds & Mammals & Flowers, Molds \\
Mammals & Birds & Flowers, Molds
\end{tabular}

Less conceptual similarity between CS+ and CS-

CS+ CS- G+ G-

Flowers Birds Molds Mammals

Molds Mammals Flowers Birds

Birds Molds Mammals Flowers

Mammals Flowers Birds Molds

Note: G+: Generalization stimuli conceptually related with

CS+, G-: Generalization stimuli conceptually related with

CS, Gu: Generalization stimuli unrelated to CS+ or CS- 
Table 2: Symptoms and breathing behavior during the acquisition trials

\begin{tabular}{lcccccc}
\hline & \multicolumn{2}{c}{ CS- } & \multicolumn{2}{c}{ CS+ Room Air } & \multicolumn{2}{c}{ CS+ $\mathrm{CO}_{2}$} \\
Variable & Mean & SE & Mean & SE & Mean & SE \\
\hline Symptom Intensity & $6.68 \mathrm{a}$ & 1.51 & $7.72 \mathrm{a}$ & 1.60 & $22.18 \mathrm{~b}$ & 1.60 \\
Symptom Unpleasantness & $6.24 \mathrm{a}$ & 1.50 & $7.03 \mathrm{a}$ & 1.60 & $22.22 \mathrm{~b}$ & 1.60 \\
Trial Valence & $2.61 \mathrm{a}$ & 0.20 & $2.76 \mathrm{a}$ & 0.21 & $4.34 \mathrm{~b}$ & 0.21 \\
Trial Arousal & $8.26 \mathrm{a}$ & 0.15 & $8.05 \mathrm{~b}$ & 0.16 & $7.04 \mathrm{c}$ & 0.16 \\
Minute Ventilation (I/min) & $8.70 \mathrm{a}$ & 0.53 & $8.72 \mathrm{a}$ & 0.54 & $10.66 \mathrm{~b}$ & 0.54 \\
FetCO & & & & & & \\
& $4.16 \mathrm{a}$ & 0.09 & $4.19 \mathrm{a}$ & 0.09 & $5.87 \mathrm{~b}$ & 0.09 \\
\hline
\end{tabular}

Note. FetCO ${ }_{2}$ : fractional end-tidal $\mathrm{CO}_{2}$

Values with different subscripts differ significantly $(p<.05)$ 


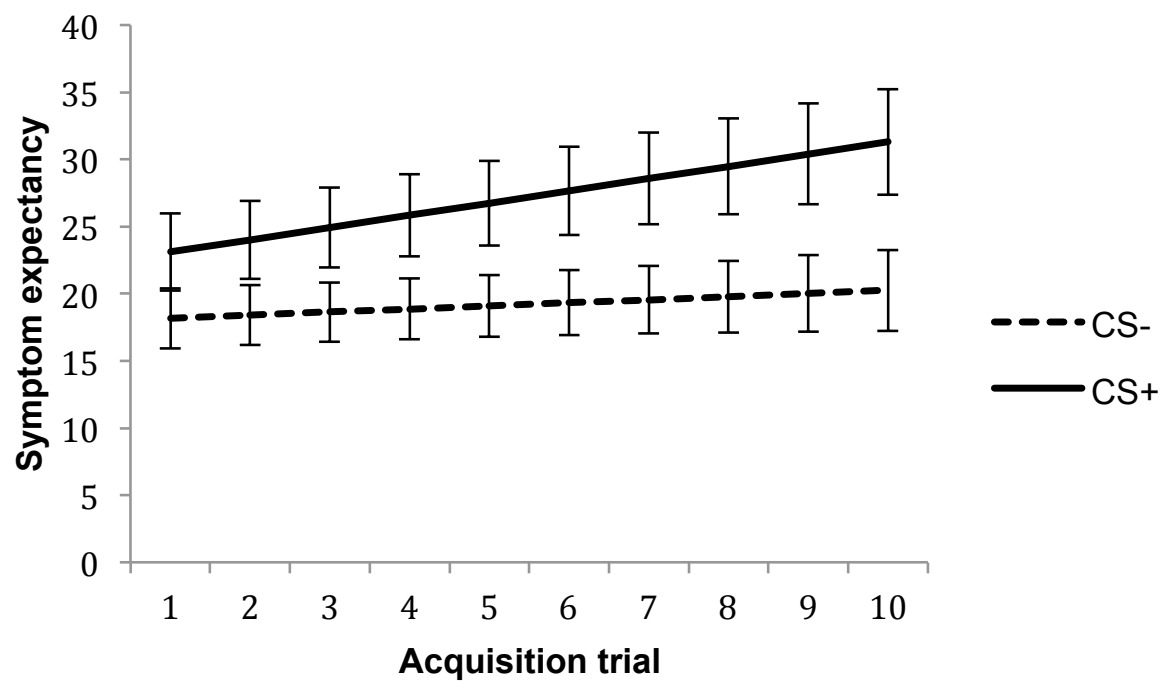

Figure 1: Acquisition of symptom expectancies (0-100 VAS Scale) over consecutive trials (Mean +-SE) 


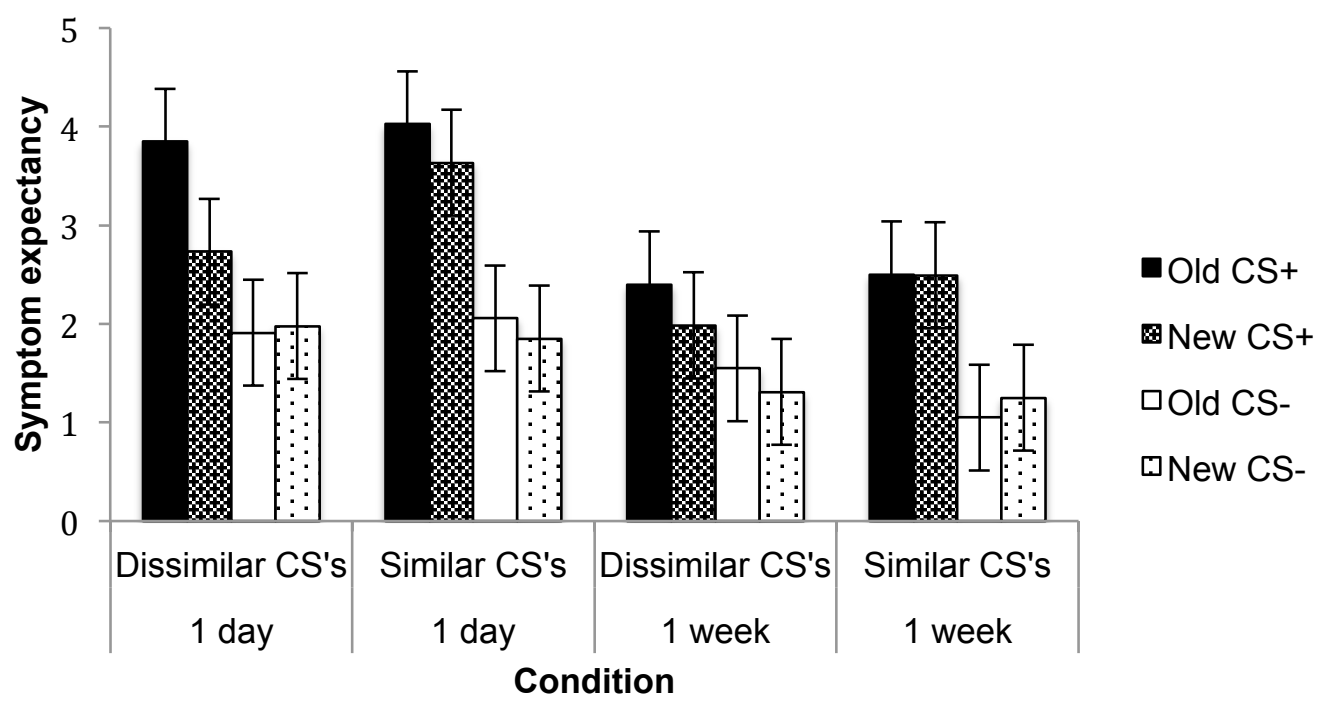

Figure 2: Impact of CS type, CS relationship, CS exemplar novelty, and timing of generalization test on symptom expectancies for old and novel CS exemplars (0-10 Scale). 


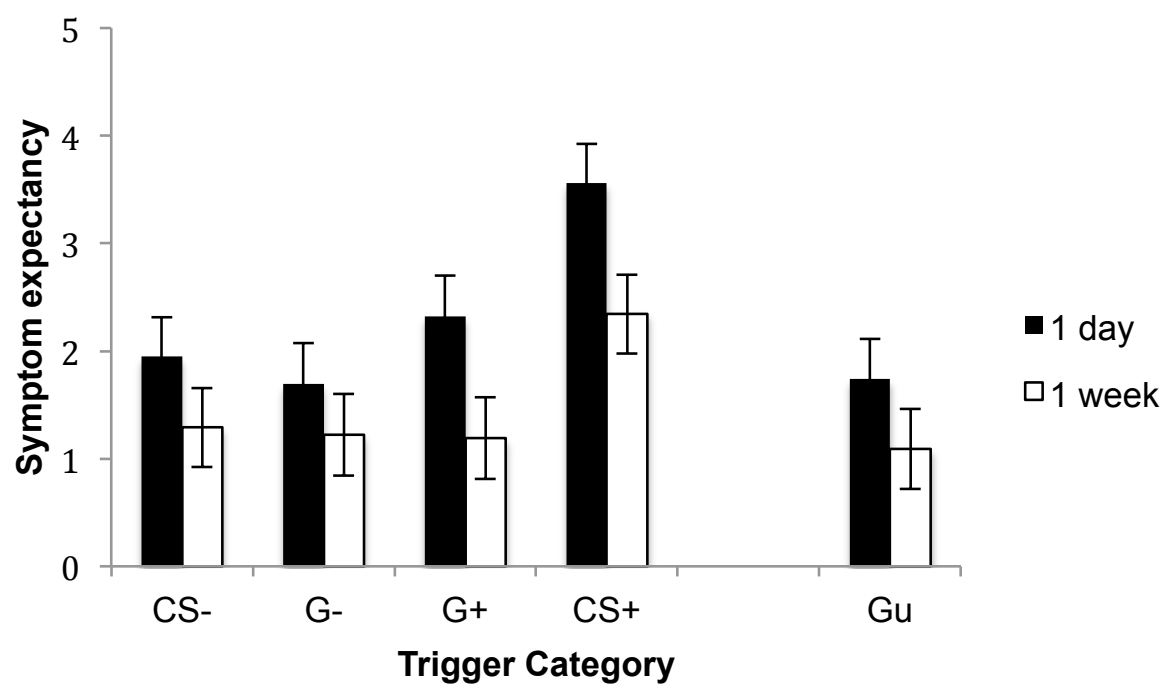

Figure 3: Generalization of symptom expectancies (0-10 Scale) to novel trigger categories, when tested 1 day or 1 week after acquisition. 